AGRICULTURE AND BIOLOGY JOURNAL OF NORTH AMERICA

ISSN Print: 2151-7517, ISSN Online: 2151-7525, doi:10.5251/abjna.2011.2.1.150.162

(C) 2011, ScienceHu $\beta$, http://www.scihub.org/ABJNA

\title{
The impact of intercropping, phosphorous addition and rhizobium inoculation on yield and nutritive value of some leguminous and cereal forages
}

\author{
Awad O. Abusuwar ${ }^{1}$ and Eltahir A. Omer ${ }^{2}$ \\ ${ }^{1}$ Dept. of Arid land Agriculture, King A/Aziz University, Jeddah, Saudi Arabia \\ E-mail: Abusuwar@yahoo.com \\ ${ }^{2}$ Faculty of Natural Resources, University of Upper Nile, Malakal, Sudan
}

\begin{abstract}
A field experiment was carried out for two consecutive seasons (2005/2006 and 2006/2007) in the Demonstration Farm of the Faculty of Agriculture at Shambat, University of Khartoum, Sudan, to study the effect of intercropping, phosphorus application and Rhizobium inoculation on the performance of some leguminous and cereal forage crops. The treatments used were pure stand, a mixture of Clitoria, lablab and Sudangrass, phosphorus fertilizer and Rhizobium inoculation. They were laid out in a completely randomized block design with three replications. Yield and quality were measured. Land equivalent ratio (LER) was calculated to evaluate intercropping versus monocropping. The results of the field experiment showed that, sole crop yield was higher than when grown in a mixture. The mixtures especially legume/ legume combinations, gave more total forage yield than the sole crops. Nearly all the intercropping treatments had land equivalent ratio (LER) above one which indicated the advantage of intercropping over monocropping. Rhizobium inoculation and phosphorus application improved forage quality by increasing crude protein content. Intercropping and phosphorus fertilization decreased crude fiber percentage in the plant tissues.
\end{abstract}

Keywords: Intercropping, nitrogen fixation, forage yield, forage quality, grass-legume mixture

\section{INTRODUCTION}

The term intercropping is usually coupled with sustaining agriculture and organic farming. Sustainability of intercropping is referring to the production of food and forage for livestock without depleting the earth resources; it is the application of nature's principles (Earles, 2005). Intercropping is becoming so important to increase crop productivity to satisfy food demands of an increasing population. Intercropping is a common cropping system in developing countries (Li et al., 1999). Nitrogen fixation is one of the most important biological processes on earth (Graham, 2008) and Rhizobium inoculation of legumes is one of the success stories of world agriculture (Herridge, 2008). Phosphorus deficiency is very common in agricultural soils and intercropping enhances phosphorus mobilization between different crop species grown in alternate rows (Li et al., 2007).

Therefore, the main objective of this study was to explore the possibility of providing green forage from an intercropping system of leguminous and nonleguminous crops with high productivity and high nutritional value. The specific objectives were to: (1) study the effect of phosphorus fertilization and Rhizobium inoculation on the growth and yield of cereal and leguminous forage crops. (2) Evaluate mixed cropping versus monocropping.

\section{MATERIALS AND METHODS}

Experimental site description: The Demonstration Farm of the Faculty of Agriculture at Shambat (latitude $15^{\circ} 40^{\prime} \mathrm{N}$ and longitude $32^{\circ} 32^{\prime} \mathrm{E}$ and altitude $280 \mathrm{~m}$ above sea level) has a semi-desert climate with annual precipitation between 100 and $200 \mathrm{~mm}$. and a temperature ranges from $12^{\circ} \mathrm{C}$ to $42^{\circ} \mathrm{C}$. (Shambat Meteorological Station,2008).

Land preparation: Four operations were carried out; ploughing, harrowing, leveling, and ridging $70 \mathrm{~cm}$ apart. The size of the plot was $3 \times 5 \mathrm{~m}$ with six ridges. The experimental plots were protected with a guard area planted with Sorghum.
Treatments
Treatments used were:
1- Sorghum sudanense (pure stand)
2- Clitoria ternatea (pure stand)
3- Lablab purpureus (pure stand)
4- Sorghum sudanense: Clitoria ternatea 
5- Sorghum sudanense: Lablab purpureus

6- Sorghum sudanense: Lablab purpureus : Clitoria ternatea

7- Sorghum sudanense + Phosphorous

8- Clitoria ternatea + Phosphorous

9- Lablab purpureus + Phosphorous

10- Sorghum sudanense: Lablab purpureus : Clitoria ternatea + Phosphorous

11- Sorghum sudanense: Lablab purpureus : Clitoria ternatea + Phosphorous + Rhizobium

12- Clitoria ternatea + Rhizobium

13- Lablab purpureus + Rhizobium

14- Clitoria ternatea : Lablab purpureus

15- Clitoria ternatea: Lablab purpureus+ Phosphorous

16- Clitoria ternatea: Lablab purpureus+ Rhizobium

17- Clitoria ternatea: Lablab purpureus+ Phosphorous+ Rhizobium

18- Sorghum sudanense: Clitoria ternatea+ Phosphorous

19- Sorghum sudanense : Clitoria ternatea+ Rhizobium

20- Sorghum sudanense: Clitoria ternatea+ Rhizobium + Phosphorous

21- Clitoria ternatea + Rhizobium + Phosphorous

22- Lablab purpureus + Rhizobium+ Phosphorous

23- Sorghum sudanense: Lablab purpureus : Clitoria ternatea+ Rhizobium

24- Sorghum sudanense : Lablab purpureus+ Phosphorous

25- Sorghum sudanense : Lablab purpureus+ Phosphorous+ Rhizobium

26- Sorghum sudanense : Lablab purpureus + Rhizobium

Treatments were replicated three times and the number of experimental plots were equal to 78 using a Randomized Complete Block Design.

Inoculation: The leguminous seeds were inoculated before planting with their Rhizobium strains (USDA3398 for Lablab and USDA3384 for Clitoria) in form of black powder, brought from the National Research Center ,Sudan.

Phosphorous addition: Phosphorous was added in the form of triple supper phosphate at a rate of $50 \mathrm{~kg}$ per hectare before planting.

Seeding rate: For sorghum, Clitoria and lablab, seeding rates applied were, 10, 15, $20 \mathrm{~kg} / \mathrm{feddan}$, respectively. In case of mixture, the seed rate was half or third of that of the sole crop (half incase of two mixtures and third incase of three mixtures).

Sowing date: The first and the second season experiments were planted on the first week of March 2005 and 2006. The first ratoons started after harvesting.

Method of planting: Seeds of legumes were drilled into the shoulders of the ridges, alternated with Sorghum seeds, row intercropping (1: 1)

Irrigation: The experiment was irrigated immediately after planting, to avoid death of bacteria ( Rhizobium ), and weekly thereafter.

Weed control: The most common weeds were Buda (Striga hermonthica), Nageela (Cynadon dactylon) and Saeda (Cyprus rotundus). Weeding was carried out once after three weeks from planting. White fly was the most common insect infested lablab plants, but did not reach threshold level.

\section{Parameters measured}

Yield: The two middle rows were harvested; each crop was separated to determine its fresh and dry weights.

Land equivalent ratio: This was determined by taking the individual crop yields in the intercropping divided by their yields as sole crops (Mead and Willey, 1980).

Nutritive value: Crude protein was determined by micro-kjeldahl method and AOAC technique (1984) was used to determine the crude fiber.

Data analysis: The Randomized Complete Block Design was used, in which the main 26 treatments were further fractionated to obtain 16,16 , and 12 treatments consisted of Clitoria, Lablab and Sorghum, respectively and each crop was statistically analyzed separately

\section{RESULTS}

Yield Clitoria ternatea

Fresh weight: The average fresh yield (tone/ha) was higher in the second season (12.6 tone/ha) compared to the first season (7 tone /ha) (Table $1 \mathrm{a}$ and $1 \mathrm{~b}$ ). Sole Clitoria, with or without addition of $P$ and $R$ significantly produced higher yield. In the first season, Clitoria in pure stand produced higher amount of green forage (15.06 tone/ha) followed by C/P/R and C/R treated plots

In the second season of 2006 (Table 1b), treatments significantly affected fresh yields of the first cut. 
Clitoria plants inoculated with Rhizobium and treated with phosphorus produced the highest fresh yield (22.22 ton/ha), whereas Clitoria grown with lablab without Rhizobium and phosphorus yielded the lowest tonnage per hector (8.7 tone/ha). Clitoria yield significantly decreased with intercropping in the two seasons.

Ratoon fresh yield was higher than the first cut yield in the two seasons (18.15 and 17.48 tone/ha for the first and second ratoon). Although fresh yield of the first ratoon was not significantly affected by treatments, Clitoria in pure stand gave higher yield (22.8 tone/ha). There was a significant difference between treatments in fresh weight in the ratoon. The CLS/P/R treatment produced the highest yield (29.4 tone/ha) followed by C/P treatment (23.2 tone/ha).

Dry weight: The response of dry weight to treatments was more or less similar to that of fresh weight. The average dry weight of Clitoria in the first cut was 1.09 and 2.91 tone/ha for the first and second year, respectively. First season dry weight showed that, the highest yield was obtained by CS/R, $\mathrm{CSL} / \mathrm{P}$ and $\mathrm{C}$ treatments. In the second season, $\mathrm{C} / \mathrm{P} / \mathrm{R}$ gave the highest dry weight (4.76 tone/ha) followed by sole Clitoria (C)

\section{Lablab purpureus}

Fresh weight: Fresh weight was significantly affected by treatments in the two seasons (Table 1a and $1 \mathrm{~b}$ ). In the first season, plots planted with sole lablab produced the highest amount of green forage per unit area (16.83 tone/ha) followed by sole lablab treated with $\mathrm{P}$ and $\mathrm{R}$ (12.58 tone/ha). Lablab in pure stand with $P$ gave the highest amount of forage per hectare (29.96) in the second season.

In the first season ratoon, L/P/R treatment also gave the highest amount of forage per unit area (11.02 tone/ha). In the second season ratoon did not show any significant difference among treatments, however, sole lablab and lablab intercropped with sorghum gave relatively higher yields.

Dry weight: Lablab /P/R gave the highest dry weight in the first cut of the two seasons (1.27 and 4.68 tone/ha for the first and the second season). In general the effect of treatments on dry weight in most treatments was similar to the effect of treatments on fresh weight, but $P$ treated plants gave slightly higher dry weight. The dry weight of the first year ratoon showed a significant difference among treatments. The L/P/R treatment resulted in the higher dry matter forage. In second season, treatments did not show any significant difference among dry weight means as in its fresh weight, but L/P, LS and LS/R treatments scored relatively high dry weight.

\section{Sorghum sudanense}

Fresh weight: As shown in tables $1 \mathrm{a}$ and $1 \mathrm{~b}$, the highest fresh yield was obtained by S/P (9.19 tone/ha) followed by sole sorghum (7.82). First crop of the second season did not show any significant difference among treatment means, but intercropped sorghum with lablab resulted in slight increase of fresh weight.

Ratoon crop showed that SCL scored the highest fresh weight in the two seasons (4.4 and 6.8 tone/ha for the first and the second year) as shown tables $1 \mathrm{a}$ and $1 b$.

Dry weight: In the first cut of the first season, the same treatment (S/P) resulted in the highest dry weight (1.94 tone/ha). There was no significant difference in dry weight of the second ratoon reading, but intercropping treatments showed a slight increase in dry weight. In ratoon crop, sorghum intercropped with legumes resulted in significantly higher dry weight of forage sorghum (Tables $1 \mathrm{a}$ and $1 \mathrm{~b}$ ).

Total yield: Total yield was significantly different in all readings of the two seasons (Table 2). In the first season, the total yield of the first cut indicated that $\mathrm{CL} / \mathrm{R}$ treatment gave the highest yield (18.43 tone/ha) followed by CSL/R. Generally intercropping legume with legume gave higher yield than sole sorghum and its combination with legumes.

Ratoon of the first year showed significant differences among treatment means and CSL/P treatment produced the highest tonnage per hectare (27.35). Clitoria with $\mathrm{P}$ plus $\mathrm{R}$ resulted in an increase in total yield. Moreover, the contribution of sorghum to the total yield was very weak.

In the first cut of the second season, lablab treated with $P$ and $R$ gave the highest yield (29.96 tone/ha). Sole Clitoria and Clitoria treated with $\mathrm{P}$ plus $\mathrm{R}$ also gave relatively higher total yield. Sorghum in general produced lower forage in the total yield.

In the second season ratoon, CSL/P/R treatment significantly scored the higher total yield (30.99 tone/ha). The three crop intercropping treatment gave more total yield than sole crops. In general sorghum was the lowest in the total yield, whereas Clitoria was the main contributor to the total forage yield.

Land equivalent ratio: The Land Equivalent Ratio for the two season cuttings were more than one, except some treatments of the first cut of the first 
season. Clitoria: Sorghum: Lablab combination kept its high ratio throughout the two seasons (Table 3).

The first cut of the first season showed high LER compared to the second cut. All the intercropping treatments had a LER grater than one (1.05 to 9.78). The lablab: Sorghum: phosphorus+Rhizobia treatment was the best one (9.78). Phosphorus alone did not show any effect on the LER.

In the first season second cut, the LER of the first crop was significantly affected by the different treatments applied. It ranged between 0.63 to 1.64 and the two intercropped treatments mainly with sorghum yielded lower than the sole crops (less than one). The higher ratio was obtained by SCL/R (1.78), followed by $\mathrm{CL}$ and CLS treatments. Sorghum: Lablab Rhizobium treatment gave the lower ratio (0.63). In the second cut (ratoon) intercropping produced more forage than sole crops. The area planted with SL/P/R and CLS yielded more than 9 times, than if cultivated by one crop. Neither Rhizobium nor $\mathrm{P}$, when applied separately, were affecting LER.

Second season of 2006 first cut showed a significant difference among the treatment means, ranging between1.06 to 3.73 which was less than the first season. Planting of the three crops together resulted in high ratios compared with the two-combinations. To some extent, second cut gave ratios similar to that of the first cut (0.99 and 6.93). Clitoria: Sorghum: Lablab treatment gave the highest ratio (6.93). Effect of $P$ was not consistent.

Crude protein (\%): Crude protein was determined at harvest in all cuttings (Tables $4 \mathrm{a}$ and $4 \mathrm{~b}$ ).

Clitoria ternatea: In the first season, first cut $\mathrm{CP}$ of Clitoria plant ranged between 16.6 to $28.33 \%$. Addition of $P$ in most cases increased the percentage of protein in the Clitoria plant.

Rhizobium inoculation, with or without $P$ increased protein content of the plant tissues. Clitoria: Lablab /P/R and $C$ treatments gave the highest percentage of CP (28.33 and 27.67 respectively).First cut of the second season showed a significant effect of treatments on crude protein. Sole Clitoria treated with $P$ and $R$ gave forage with the highest amount of protein (25.08) in this reading. Intercropping with sorghum reduced $\mathrm{CP} \%$ in Clitoria.

In the second cut, treatments significantly affected crude protein of Clitoria. Crude protein ranged between 15.67 to $26.33 \%$. Intercropping legume with legume $(\mathrm{CL})$ and addition of $\mathrm{P}$ increased crude protein. Clitoria treated with $\mathrm{P}$ and inoculated with $\mathrm{R}$ produced plants with the highest CP \% (26.33) followed by CL/R combination (24\%).

In the second season, means of crude protein of Clitoria were significantly affected by treatments. The $\mathrm{CP}$ ranged between 15.17 and $25.08 \%$. Intercropping of the two plants gave slightly better crude protein than the intercropping of the three crops.

Second season ratoon showed a significant difference among treatments in crude protein of Clitoria. Clitoria inoculated with $\mathrm{R}$ produced forage with high crude protein (24\%), whereas Clitoria intercropped with sorghum gave forage with low nutritional value (CP of 15.18\%).

Lablab purpureus: In the first season, the first cut produced plants with $\mathrm{CP}$ ranged between 10 and $15 \%$. Sole lablab inoculated with $\mathrm{R}$ and sole lablab treated with $\mathrm{P}$ and $\mathrm{R}$ gave forage with high CP\% (15). In general $\mathrm{R}$ inoculation and/or $\mathrm{P}$ addition increased the percentage of $\mathrm{CP}$.

In the first cut of the second season, L/P/R produced forage with high quality (17.08\% protein). Inoculation of two-combination intercropped plots resulted in relatively high $\mathrm{CP} \%$.

In the ratoon crop of lablab, crude protein was significantly different in the two seasons. Lablab grown with Clitoria and treated with $\mathrm{P}$ and $\mathrm{R}$ gave the highest CP\% in the first year ratoon, whereas the lowest CP\% (10.5) was observed in lablab grown with sorghum (LS). Lablab treated with $\mathrm{P}$ expressed a high percentage of CP.

In the second season ratoon, lablab grown alone and inoculated with $R$ resulted in higher $C P$. Addition of $P$ and $\mathrm{R}$ significantly increased the $\mathrm{CP}$.

Sorghum sudanense: Crude protein of Sudangrass was significantly affected by the different treatments in all cuttings. In the first cut of the first season, CP\% ranged between 6.5 and 11.8 . It was clearly observed that intercropping sorghum with legume significantly increased the CP of sorghum. Moreover, addition of $\mathrm{P}$ to the sorghum increased the amount of CP. Sorghum alone scored the lowest amount of CP (6.5).

In the second season, first cut showed significant differences between means of crude protein due to the application of different treatments. Sole sorghum gave the lowest CP (6.75), whereas sorghum intercropped with Clitoria and treated with $P$ resulted in higher percentage of CP. Addition of phosphorus increased the crude protein of sorghum forage. 
Agric. Biol. J. N. Am., 2011, 2(1): 150-162

Table1a Effect of intercropping, phosphorus and inoculation on fresh and dry yield (t/ha) of Clitoria, lablab and sorghum (2005)

\begin{tabular}{|c|c|c|c|c|c|c|c|c|c|c|c|c|}
\hline \multirow{3}{*}{ Treatment } & \multicolumn{4}{|c|}{ Clitoria } & \multicolumn{4}{|c|}{ Lablab } & \multicolumn{4}{|c|}{ Sorghum } \\
\hline & \multicolumn{2}{|c|}{$1^{\text {st }}$ harvest } & \multicolumn{2}{|c|}{$2^{\text {nd }}$ harvest } & \multicolumn{2}{|c|}{$1^{\text {st }}$ harvest } & \multicolumn{2}{|c|}{$2^{\text {nd }}$ harvest } & \multicolumn{2}{|c|}{$1^{\text {st }}$ harvest } & \multicolumn{2}{|c|}{$2^{\text {nd }}$ harvest } \\
\hline & Fresh wt & Dry wt & Fresh wt & Dry wt & Fresh wt & Dry wt & Fresh wt & Dry wt & Fresh wt & Dry wt & Fresh wt & Dry wt \\
\hline$L / P / R$ & & & & & $12.58 \mathrm{ab}$ & $1.27 a$ & $11.02 \mathrm{a}$ & $1.697 a$ & & & & \\
\hline $\mathrm{SCL}$ & $4.803 \mathrm{BC}$ & $0.99 a b$ & $51.31 \mathrm{a}$ & $3.52 \mathrm{cde}$ & $6.05 a b$ & $0.65 a b$ & $0.48 c$ & $0.13 \mathrm{bc}$ & $3.06 \mathrm{bc}$ & $0.92 \mathrm{ab}$ & $4.42 a$ & $0.68 \mathrm{ab}$ \\
\hline $\mathrm{SC} / \mathrm{P} / \mathrm{R}$ & $5.71 b c$ & $0.31 b$ & 22.12 & $5.76 a b c$ & & & & & $4.59 \mathrm{bc}$ & $0.61 a b$ & 0.85 & 0.34 \\
\hline $\mathrm{SL}$ & & & & & $4.49 \mathrm{~b}$ & $0.42 a b$ & $2.83 \mathrm{bc}$ & $0.40 \mathrm{bc}$ & $2.21 \mathrm{c}$ & $1.22 \mathrm{ab}$ & $1.02 \mathrm{a}$ & $0.26 \mathrm{~b}$ \\
\hline $\mathrm{SL} / \mathrm{R}$ & & & & & $2.89 b$ & $0.20 \mathrm{~b}$ & $8.77 a b$ & $0.997 a b c$ & $2.21 \mathrm{c}$ & $0.06 a b$ & $0.87 a$ & $0.13 \mathrm{~b}$ \\
\hline SL/P & & & & & 6.32ab & $1.02 \mathrm{ab}$ & 9.35ab & $0.9 a b c$ & $4.83 \mathrm{bc}$ & $0.99 a b$ & $3.12 a$ & 0.58 \\
\hline SL/P/R & & & & & $4 . .83 a$ & $0.78 a b$ & $6.12 \mathrm{abc}$ & 1.08abc & $4.08 \mathrm{bc}$ & 1.43 & $3.86 a$ & $0.78 a b$ \\
\hline $\mathrm{L}$ & & & & & $16.83 a$ & $0.7 a b$ & $5.95 a b c$ & $0.78 a b c$ & & & & \\
\hline L/P & & & & & $10.24 a b$ & $1.09 a b$ & 7.27abc & 1.44ab & & & & \\
\hline $\mathrm{s}$ & & & & & & & & & 7.82ab & $1.49 a b$ & $1.50 a$ & $0.68 a b$ \\
\hline SCL/P/R & $2.397 \mathrm{c}$ & $0.34 b$ & $11.7 \mathrm{a}$ & 3.44cde & $3.06 \mathrm{~b}$ & $0.34 a b$ & $1.02 \mathrm{c}$ & 0.07 & $2.11 \mathrm{c}$ & $0.34 b$ & $1.63 a$ & $0.577 a b$ \\
\hline L/R & & & & & $9.18 a b$ & $1.19 a b$ & $6.80 a b c$ & $0.91 \mathrm{abc}$ & & & & \\
\hline $\mathrm{SCL} / \mathrm{R}$ & $5.66 \mathrm{bc}$ & 1.77ab & $13.75 a$ & $3.23 \mathrm{de}$ & 7.65ab & $1.05 \mathrm{ab}$ & $1.19 c$ & 0.47 & $4.08 \mathrm{bc}$ & $1.26 a b$ & $2.42 a$ & $0.71 \mathrm{ab}$ \\
\hline $\mathrm{SCL} / \mathrm{P}$ & $3.60 \mathrm{cb}$ & $0.85 a b$ & $21.6 a$ & $4.05 \mathrm{bcd}$ & $4.42 b$ & $0.68 a b$ & 0.51 & $0.03 c$ & $2.62 \mathrm{c}$ & $0.85 a b$ & $5.27 a$ & $0.30 a$ \\
\hline $\mathrm{C} / \mathrm{P}$ & $8.57 \mathrm{bc}$ & 1.43ab & $18.56 a$ & $6.25 \mathrm{an}$ & & & & & & & & \\
\hline C/P/R & $9.70 \mathrm{ab}$ & $1.50 \mathrm{ab}$ & $20.2 a$ & 4.54abcd & & & & & & & & \\
\hline SC & $4.19 \mathrm{bc}$ & $0.92 a$ & $12.08 \mathrm{a}$ & $2.87 \mathrm{e}$ & & & & & $4.13 b c$ & $0.54 b$ & $2.55 a$ & $0.88 a$ \\
\hline $\mathrm{SC} / \mathrm{R}$ & $7.55 \mathrm{bc}$ & $1.97 a$ & $22.5 a$ & $5.30 a b c$ & & & & & $3.40 \mathrm{bc}$ & $1.02 \mathrm{ab}$ & $0.92 a$ & $0.36 a b$ \\
\hline $\mathrm{SC} / \mathrm{P}$ & $4.80 \mathrm{~b}$ & $1.36 \mathrm{ab}$ & $15.99 a$ & $\begin{array}{c}\text { 5.01abcd } \\
\mathrm{e} \\
\end{array}$ & & & & & $4.08 \mathrm{bc}$ & $0.15 a b$ & $0.55 a$ & $0.33 a b$ \\
\hline $\mathrm{S} / \mathrm{P}$ & & & & & & & & & $9.19 a$ & $0.94 a$ & $0.85 a$ & $0.51 \mathrm{ab}$ \\
\hline C & $15.06 \mathrm{a}$ & 1.73ab & $22.8 a$ & $6.75 a$ & & & & & & & & \\
\hline $\mathrm{CL} / \mathrm{P}$ & $6.77 \mathrm{bc}$ & 095ab & $15.8 a$ & 5.107 & $4.42 \mathrm{~b}$ & $0.48 a b$ & $0.4 \mathrm{c}$ & $0.52 \mathrm{abc}$ & & & & \\
\hline $\mathrm{CL}$ & $7.04 \mathrm{bc}$ & $0.88 a b$ & $16.50 \mathrm{a}$ & $\begin{array}{c}\text { 5.10abcd } \\
\mathrm{e}\end{array}$ & 7.99ab & $0.48 a b$ & $0.27 c$ & $0.17 c$ & & & & \\
\hline $\mathrm{C} / \mathrm{R}$ & $9.60 \mathrm{ab}$ & $1.33 a b$ & $20.9 a$ & $6.42 \mathrm{ab}$ & & & & & & & & \\
\hline $\mathrm{CL} / \mathrm{R}$ & $7.89 \mathrm{bc}$ & 054ab & $20.5 a$ & $6.79 a$ & $10.56 \mathrm{ba}$ & $0.48 a b$ & $4.25 a b c$ & $0.907 a b c$ & & & & \\
\hline $\mathrm{CL} / \mathrm{P} / \mathrm{R}$ & $8.58 \mathrm{bc}$ & 0.61 & 20.05 & 5.43abcd & $6.80 \mathrm{ab}$ & $0.95 a b$ & $3.88 \mathrm{bc}$ & $0.81 \mathrm{abc}$ & & & & \\
\hline $\mathrm{SE} \pm$ & 0.48 & 0.01 & 0.998 & 0.18 & 0.81 & 0.07 & 0.52 & 0.09 & 0.38 & 0.11 & 0.47 & 0.04 \\
\hline $\mathrm{CV}$ & 47.4 & 70.25 & 35.1 & 24.9 & 76.1 & 70.4 & 81.4 & 83.4 & 58.66 & 65.24 & 137.2 & 55.3 \\
\hline
\end{tabular}

Means with the same letter (s) within each column are not significantly different at 0.05 level using Duncan's Multiple Range Test.

Legend: $\mathrm{C}=$ Clitoria, $\mathrm{L}=$ lablab, $\mathrm{S}=$ sorghum, $\mathrm{P}=$ phosphors, $\mathrm{R}=$ Rhizobium 
Agric. Biol. J. N. Am., 2011, 2(1): 150-162

Table 1b Effect of intercropping, phosphorus and inoculation on fresh and dry yield ( $\mathrm{t} / \mathrm{ha}$ ) of Clitoria, lablab and sorghum (2006)

\begin{tabular}{|c|c|c|c|c|c|c|c|c|c|c|c|c|}
\hline \multirow{3}{*}{ Treatment } & \multicolumn{4}{|c|}{ Clitoria } & \multicolumn{4}{|c|}{ Lablab } & \multicolumn{4}{|c|}{ Sorghum } \\
\hline & \multicolumn{2}{|c|}{$1^{\text {st }}$ harvest } & \multicolumn{2}{|c|}{$2^{\text {nd }}$ harvest } & \multicolumn{2}{|c|}{$1^{\text {st }}$ harvest } & \multicolumn{2}{|c|}{$2^{\text {nd }}$ harvest } & \multicolumn{2}{|c|}{$1^{\text {st }}$ harvest } & \multicolumn{2}{|c|}{$2^{\text {nd }}$ harvest } \\
\hline & Fresh wt & Dry wt & Fresh wt & Dry wt & Fresh wt & Dry wt & Fresh wt & Dry wt & Fresh wt & Dry wt & Fresh wt & Dry wt \\
\hline$L / P / R$ & & & & & $29.96 a$ & $4.68 a$ & $0.66 a$ & $0.66 a$ & & & & \\
\hline $\mathrm{SCL}$ & $4.25 d$ & $1.11 d$ & $15.9 a b$ & $4.37 a$ & $8.02 b$ & $1.53 a b$ & $1.99 a$ & $0.55 a$ & $0.49 a$ & $2.38 a$ & $6.75 a$ & $2.51 a$ \\
\hline $\mathrm{SC} / \mathrm{P} / \mathrm{R}$ & 13.89abcd & $3.49 a b c$ & 18.1 & 4.09 & & & & & $8.75 a$ & 2.7 & $1.39 \mathrm{abc}$ & $0.44 b$ \\
\hline SL & & & & & $5.72 \mathrm{~b}$ & $1.27 \mathrm{~b}$ & $6.15 a$ & $1.13 a$ & $8.29 a$ & $2.68 a$ & $1.39 a b c$ & $0.44 b$ \\
\hline $\mathrm{SL} / \mathrm{R}$ & & & & & $7.74 b$ & $2.1 \mathrm{ab}$ & $4.47 a$ & $1.22 \mathrm{a}$ & $6.74 a$ & $2.10 \mathrm{a}$ & $3.57 a b c$ & $1.37 a b$ \\
\hline SL/P & & & & & $9.40 b$ & $2.49 a b$ & $1.39 a$ & $0.28 a$ & $4.17 a$ & $2.10 a$ & $5.83 a b c$ & $1.86 a b$ \\
\hline SL/P/R & & & & & $4.44 b$ & $1.43 a$ & $2.98 a$ & $0.78 a$ & $4.17 a$ & $1.35 a$ & $6.35 a b$ & $1.55 a b$ \\
\hline $\mathrm{L}$ & & & & & $11.71 \mathrm{~b}$ & $2.17 a b$ & $4.17 a$ & 0.36 & $5.52 a$ & $1.71 \mathrm{a}$ & 1.99abc & $0.65 b$ \\
\hline $\mathrm{L} / \mathrm{P}$ & & & & & $13.89 \mathrm{~b}$ & $2.54 a b$ & $3.13 a$ & $1.04 a$ & & & & \\
\hline $\mathrm{s}$ & & & & & & & & & $3.97 a$ & $1.39 a$ & $2.45 a b c$ & $1.57 a b$ \\
\hline $\mathrm{SCL} / \mathrm{P} / \mathrm{R}$ & 11.31 & $2.5 \mathrm{abcd}$ & $29.4 a$ & $6.07 a$ & $1.19 b$ & $1.67 a b$ & $2.09 a$ & $0.30 a$ & $3.89 a$ & $1.15 a$ & $0.71 b c$ & $0.60 \mathrm{~b}$ \\
\hline L/R & & & & & 16.ab & $2.62 \mathrm{ab}$ & $3.55 a$ & 1.24 & & & & \\
\hline $\mathrm{SCL} / \mathrm{R}$ & $7.94 \mathrm{~cd}$ & $1.63 \mathrm{dc}$ & $16.3 a b$ & $3.77 a$ & $3.56 \mathrm{~b}$ & $0.48 a$ & $1.28 \mathrm{a}$ & $0.27 a$ & $71.30 \mathrm{a}$ & $3.14 a$ & $0.75 b c$ & $0.20 \mathrm{~b}$ \\
\hline $\mathrm{SCL} / \mathrm{P}$ & $1.71 \mathrm{bcd}$ & 3..55abc & $17.5 a b$ & $5.55 a$ & 2.98 & $0.30 \mathrm{~b}$ & $1.79 a$ & $0.36 a$ & $8.5 a$ & $1.82 \mathrm{a}$ & 1.79abc & $0.67 b$ \\
\hline $\mathrm{C} / \mathrm{P}$ & $17.26 \mathrm{abc}$ & $3.55 a b c$ & $23.2 \mathrm{ab}$ & $4.89 a$ & & & & & & & & \\
\hline $\mathrm{C} / \mathrm{P} / \mathrm{R}$ & $22.22 a$ & $4.76 \mathrm{a}$ & $16.5 a b$ & $5.32 a$ & & & & & & & & \\
\hline $\mathrm{SC}$ & $10.52 \mathrm{bcd}$ & 2.66abcdf & $19.4 a b$ & $4.75 a$ & & & & & $5.95 a$ & $.67 a$ & $0.60 c$ & $0.14 b$ \\
\hline $\mathrm{SC} / \mathrm{R}$ & 11.3bcd & 2.76abcd & 15.5 & $3.93 a$ & & & & & 6.35 & $1.46 a$ & $0.60 c$ & $0.60 \mathrm{~b}$ \\
\hline $\mathrm{SC} / \mathrm{P}$ & $11.03 \mathrm{bcd}$ & $2.76 \mathrm{abcd}$ & $14.3 a b$ & 3.37 & & & & & $2.19 a$ & $0.48 a$ & 0.42 & $0.10 a$ \\
\hline $\mathrm{S} / \mathrm{P}$ & & & & & & & & & $4.76 a$ & $1.15 a$ & $5.82 \mathrm{abc}$ & $1.71 \mathrm{ab}$ \\
\hline$C$ & 18.097ab & $4.36 \mathrm{a}$ & $17.7 a b$ & $5.72 a$ & & & & & & & & \\
\hline $\mathrm{CL} / \mathrm{P}$ & 16.27abc & 3.06abcd & $14.7 \mathrm{ab}$ & $4.25 a$ & $2.9 \mathrm{~b}$ & $0.66 \mathrm{~b}$ & $2.68 a$ & $0.51 \mathrm{a}$ & & & & \\
\hline $\mathrm{CL}$ & $8.7 \mathrm{bcd}$ & $1.98 \mathrm{bcd}$ & $15.9 a b$ & $6.35 a$ & $9.53 b$ & $1.62 \mathrm{ab}$ & $1.44 a$ & 0.61 & & & & \\
\hline $\mathrm{C} / \mathrm{R}$ & 17.86ab & 4.09ab & $14.9 a b$ & $6.25 a$ & & & & & & & & \\
\hline $\mathrm{CL} / \mathrm{R}$ & 9.087 & 3.3abcd & 13.7 & $6.13 a$ & $9.72 b$ & $2.29 a b$ & $0.66 a$ & $0.13 a$ & & & & \\
\hline $\mathrm{CL} / \mathrm{P} / \mathrm{R}$ & $9.05 \mathrm{bcd}$ & 2.66abcdf & $16.7 a b$ & 5.22 & $12.30 \mathrm{~b}$ & $2.25 \mathrm{ab}$ & $0.66 a$ & $0.61 \mathrm{a}$ & & & & \\
\hline $\mathrm{SE} \pm$ & 0.72 & 0.17 & 1.1 & 0.38 & 1.1 & 2.21 & 0.43 & 0.09 & 0.6 & 0.22 & 0.38 & 0.12 \\
\hline $\mathrm{CV}$ & 39.54 & 41.2 & 43.6 & 52.04 & 74.97 & 74.88 & 118.4 & 105.4 & 6.8 & 83.7 & 78.7 & 70.7 \\
\hline
\end{tabular}

Means with the same letter (s) within each column are not significantly different at 0.05 level using Duncan's Multiple Range Test.

Legend: $\mathrm{C}=$ Clitoria, $\mathrm{L}=$ lablab, $\mathrm{S}=$ sorghum, $\mathrm{P}=$ phosphors, $\mathrm{R}=$ Rhizobium . 
Agric. Biol. J. N. Am., 2011, 2(1): 150-162

Table 2 Effect of intercropping, phosphorus and inoculation on total yield (t/ha) of Clitoria, lablab and sorghum (2005 and 2006)

\begin{tabular}{|c|c|c|c|c|}
\hline \multirow{2}{*}{ Treatment } & \multicolumn{2}{|c|}{ First season } & \multicolumn{2}{|c|}{ Second season } \\
\hline & $1^{\text {st }}$ harvest & $2^{\text {nd }}$ harvest & $1^{\text {st }}$ harvest & $2^{\text {nd }}$ harvest \\
\hline CSL/P/R & $7.6 a b$ & 14.4 bcde & 15.7 bcde & $30.99 a$ \\
\hline CSL/P & $10.6 \mathrm{ab}$ & $27.35 \mathrm{a}$ & $21.2 \mathrm{abcd}$ & $20.4 \mathrm{abcd}$ \\
\hline $\mathrm{CL}$ & $15.03 \mathrm{ab}$ & $16.8 \mathrm{abcd}$ & 15.1 bcde & 16.9 bcdef \\
\hline $\mathrm{CS} / \mathrm{P}$ & $8.88 \mathrm{ab}$ & 16.5 abcde & 15.6bcde & 14.6 bcdefgh \\
\hline C & $15.1 \mathrm{ab}$ & $22.8 \mathrm{abc}$ & $18.1 \mathrm{bc}$ & 17.4 bcdefg \\
\hline $\mathrm{CS} / \mathrm{R}$ & $10.92 \mathrm{ab}$ & $23.4 \mathrm{abc}$ & $17.4 \mathrm{bc}$ & 12.7 bcdefghi \\
\hline $\mathrm{CS} / \mathrm{P} / \mathrm{R}$ & $10.3 \mathrm{ab}$ & $22.96 a b c$ & $22.6 \mathrm{ab}$ & 19.5 abcde \\
\hline CSL/R & $17.2 \mathrm{ab}$ & $17.36 \mathrm{abc}$ & $15.96 \mathrm{bcd}$ & 18.25 abcdef \\
\hline$C / R$ & $9.6 a b$ & $20.9 a b c$ & $17.86 \mathrm{bc}$ & 7.34 defghi \\
\hline $\mathrm{C} / \mathrm{P}$ & $8.57 \mathrm{ab}$ & $17.57 \mathrm{abcd}$ & $17.26 \mathrm{bc}$ & $23.2 \mathrm{abc}$ \\
\hline $\mathrm{CS}$ & $8.37 \mathrm{ab}$ & 14.36 abcde & $16.74 \mathrm{bcd}$ & $19.85 \mathrm{abcd}$ \\
\hline $\mathrm{C} / \mathrm{P} / \mathrm{R}$ & $9.7 \mathrm{ab}$ & $20.2 \mathrm{abc}$ & $22.2 \mathrm{abc}$ & 16.5 bcdefg \\
\hline CSL & $13.36 a b$ & $20.2 \mathrm{abc}$ & $20.8 a b c$ & $24.61 \mathrm{ab}$ \\
\hline $\mathrm{CL} / \mathrm{P}$ & $11.37 \mathrm{ab}$ & $16.64 \mathrm{abcd}$ & $19.18 \mathrm{abc}$ & 16.14 bcdefg \\
\hline $\mathrm{CL} / \mathrm{R}$ & $18.43 \mathrm{a}$ & $24.75 a b$ & $18.81 \mathrm{abc}$ & 14.37 bcdefgh \\
\hline $\mathrm{CL} / \mathrm{P} / \mathrm{R}$ & $15.4 \mathrm{ab}$ & $20.6 \mathrm{abc}$ & $21.35 a b c$ & 17.1 bcdefg \\
\hline SL/P/R & $9.91 \mathrm{ab}$ & 11 cdef & 06.96 cde & $4.96 \mathrm{fghi}$ \\
\hline $\mathrm{S}$ & 7. $82 \mathrm{ab}$ & $1.5 \mathrm{f}$ & $3.97 \mathrm{e}$ & $2.45 \mathrm{hi}$ \\
\hline SL/R & $5.07 \mathrm{~b}$ & 10.6 cdef & 11.9 bcde & 8.9 defghi \\
\hline $\mathrm{S} / \mathrm{P}$ & $8.53 \mathrm{ab}$ & $0.85 f$ & $4.76 \mathrm{de}$ & 5.8 efghi \\
\hline SL/P & $11.15 \mathrm{ab}$ & 12.5 bcdef & 13.6bcde & 8.02 defghi \\
\hline $\mathrm{SL}$ & $6.7 \mathrm{ab}$ & $3.85 \mathrm{fe}$ & 14 bcde & 9.7 cdefghi \\
\hline L/P/R & $12.6 \mathrm{ab}$ & 11.0 cdef & $29.96 \mathrm{a}$ & $0.44 \mathrm{i}$ \\
\hline$L / R$ & $9.18 \mathrm{ab}$ & 6.8 def & $16.1 \mathrm{bcd}$ & $2.36 \mathrm{hi}$ \\
\hline $\mathrm{L}$ & $16.8 \mathrm{ab}$ & 5.95 def & 11.7 bcde & $4.17 \mathrm{ghi}$ \\
\hline L/P & $10.54 \mathrm{ab}$ & 7.27 def & 13.9 bcde & $2.09 \mathrm{hi}$ \\
\hline SE & 6.1 & 6.5 & 6.11 & 6.9 \\
\hline $\mathrm{CV}$ & 55.3 & 43.7 & 37.3 & 53 \\
\hline
\end{tabular}

Means with the same letter (s) within each column are not significantly different at 0.05 level using Duncan's Multiple Range Test. Legend: $\mathrm{C}=$ Clitoria, $\mathrm{L}=$ lablab, $\mathrm{S}=$ sorghum, $\mathrm{P}=$ phosphors, $\mathrm{R}=$ Rhizobium 
Agric. Biol. J. N. Am., 2011, 2(1): 150-162

Table 3 Effect of intercropping, phosphorus and inoculation on land equivalent ratio for Clitoria, lablab and Sorghum

\begin{tabular}{|c|c|c|c|c|}
\hline \multirow{2}{*}{ Treatment } & \multicolumn{2}{|c|}{ First season } & \multicolumn{2}{|c|}{ Second season } \\
\hline & $1^{\text {st }}$ harvest & $2^{\text {nd }}$ harvest & 1st harvest & $2^{\text {nd }}$ harvest \\
\hline $\mathrm{SCL}$ & 9.653 & 1.301 & 2.707 & 6.93 \\
\hline $\mathrm{SC} / \mathrm{P} / \mathrm{R}$ & 4.09 & 0.897 & 2.93 & 2.82 \\
\hline $\mathrm{SL}$ & 1.337 & 0.727 & 2.96 & 3.934 \\
\hline $\mathrm{SL} / \mathrm{R}$ & 3.607 & 0.634 & 2.6 & 3.25 \\
\hline SL/P & 6.827 & 1.597 & 2.33 & 3.484 \\
\hline $\mathrm{SL} / \mathrm{P} / \mathrm{R}$ & 9.836 & 1.05 & 2.08 & 1.473 \\
\hline SCL/P/R & 3.104 & 0.729 & 1.736 & 2.716 \\
\hline SCL/R & 4.033 & 1.783 & 2.457 & 2.164 \\
\hline SCL/P & 3.051 & 1.223 & 3.73 & 2.407 \\
\hline $\mathrm{SC}$ & 4.377 & 0.893 & 2.403 & 1.673 \\
\hline $\mathrm{SC} / \mathrm{R}$ & 1.75 & 0.923 & 2.077 & 1.29 \\
\hline $\mathrm{SC} / \mathrm{P}$ & 1.713 & 0.893 & 1.44 & 1 \\
\hline $\mathrm{CL} / \mathrm{P}$ & 1.05 & 1.073 & 1.363 & 1.247 \\
\hline $\mathrm{CL}$ & 1.097 & 1.297 & 1.063 & 1.284 \\
\hline $\mathrm{CL} / \mathrm{R}$ & 1.93 & 1.674 & 1.54 & 0.997 \\
\hline $\mathrm{CL} / \mathrm{P} / \mathrm{R}$ & 1.746 & 1.003 & 1.813 & 1.134 \\
\hline SD & 2.81 & 0.18 & 0.49 & 2.3 \\
\hline
\end{tabular}

Means with the same letter (s) within each column are not significantly different at 0.05 level using Duncan's Multiple Range Test.

Legend: $\mathrm{C}=$ Clitoria, $\mathrm{L}=$ lablab, $\mathrm{S}=$ sorghum, $\mathrm{P}=$ phosphors, $\mathrm{R}=$ Rhizobium

The crude protein in first season ratoon significantly increased with the intercropping and $P$ application. In this cut, SC/P/R treatment gave plants with high CP (11\%), whereas sole sorghum was significantly lower in CP.

In the second season ratoon, the CP\% ranged between 7.5 and 11.5. Generally, intercropping increased CP\%. Sorghum grown with Clitoria, lablab and treated with $P$ and $R$ gave forage with high amount of CP (11.5).

\section{Crude fiber (\%)}

Clitoria ternatea: Crude fiber of Clitoria was significantly affected by the different treatments applied in all cuttings (Table $5 a$ and 5b).

In the first season, crude fiber of the first cut significantly decreased by intercropping and addition of $P$. Crude fiber of the forage ranged 25.75 and $34.18 \%$. It was also observed that addition of $P$ and $\mathrm{R}$ to the Clitoria intercropped with the other legume (lablab) decreased the crude fiber of Clitoria. Sole Clitoria had the highest CF\% (34.18).
In the first cut of the second season, Clitoria grown alone recorded the highest CP\% (32.73), whereas CL/R got the lowest percentage (25.28)

Crude fiber of Clitoria in the first season ratoon was significantly affected by intercropping and addition of $P$. Plots grown with Clitoria alone produced more fibrous forage $(33.78 \%)$ than the mixture and in most cases addition of $\mathrm{P}$ and $\mathrm{R}$ to the plants decreased CF\%.

For the second season ratoon, also sole crops gave forage with high CF\% (30.5). Addition of $P$ and $R$ was observed to reduce CF compared to intercropping Clitoria with sorghum.

Lablab purpureus: Crude fiber of lablab CF was significantly affected in all cuttings due to the application of different treatments.

The first cut of the first season produced forage with CF ranged between 30.48 and 39.08. Lablab grown alone was significantly fibrous than the intercropped one. The treatments $\mathrm{L}$ and LS resulted in the higher CF forage (39\%), whereas LSC /R treatment produced plants with the lower CF\%.

In the first cut of the second season, sole lablab plants had the higher CF\% (39.08), whereas LSC/R 
gave the lowest CF\% (30.1). Generally $\mathrm{P}$ and $\mathrm{R}$ decreased CF\%.

Crude fiber of ratoon of the first season was relatively higher in CF than the first cut. Commonly sole lablab produced forage with higher $\mathrm{CF}$ and intercropping treatments with the addition of $P$ and $R$ significantly decreased the amount of fiber in the lablab forage. In many readings, LC/P/R treatment gave the lowest CF forage.

Ratoon in the second season ratoon revealed lower CF\% (25.76 to $32.2 \%$ ) compared with ratoon of the first season. Plots with LS/P produced forage with higher CF \%( 34), whereas LC/P/R treatment gave forage lower in CF (25.76\%).

Sorghum sudanense: Crude fiber of sorghum plant was significantly affected by treatments in the two seasons. The CF\% ranged between 35.94 and 42.2 in the first cut of the first season. Sorghum interplanted with Clitoria, addition of $\mathrm{P}$ and $\mathrm{R}$ gave the lower CF. Addition of $\mathrm{P}$ alone to the sorghum did not significantly increase the CF. In the first cut of the second season, CF ranged between 37.06 and 44.7. The treatment SLC/P/R gave plants with the lower CF (37.06), whereas S/P treatment forage sorghum with high CF (44.7)

The first year ratoon gave forage with 30.24 to $41 \%$ CF. Sole sorghum plants obtained the highest CP\% (41.68), whereas SCL/P/R gave the lowest CF forage sorghum (30.24).Intercropping and addition of $P$ was observed to decrease CF.

The second year ratoon produced sorghum forage with CF ranging between 33.5 and 41.03. Sole sorghum plant was more fibrous (41.03) than the other sorghum which planted with legumes as intercropping. In general intercropping gave plants with lower CF.

Table 4a. Effect of intercropping, phosphorus and inoculation on crude protein (\%) of Clitoria, lablab and sorghum (2005)

\begin{tabular}{|c|c|c|c|c|c|c|}
\hline \multirow[t]{2}{*}{ Treatment } & \multicolumn{2}{|l|}{ Clitoria } & \multicolumn{2}{|l|}{ Lablab } & \multicolumn{2}{|l|}{ Sorghum } \\
\hline & At $1^{\text {st }}$ harvest & $\begin{array}{l}\text { At } 2^{\text {nd }} \\
\text { harvest }\end{array}$ & At $1^{\text {st }}$ harvest & At $2^{\text {nd }}$ harvest & $\begin{array}{c}\text { At } 1^{\text {st }} \\
\text { harvest }\end{array}$ & At $2^{\text {nd }}$ harvest \\
\hline $\mathrm{L} / \mathrm{P} / \mathrm{R}$ & & & $15 a$ & $12.33 \mathrm{bcd}$ & & \\
\hline SCL & $19.5 \mathrm{cdef}$ & 17.efg & 10.67def & $11.83 a$ & $10.17 a b$ & $7.83 d$ \\
\hline $\mathrm{SC} / \mathrm{P} / \mathrm{R}$ & $17.83 \mathrm{fg}$ & 18.33defg & & & $11.83 a$ & $11 a$ \\
\hline SL & & & 11.17cdef & $10.5 d$ & $11 a b$ & 10 \\
\hline SL /R & & & 10.17ef & 12.bcd & $10.33 a b$ & $9.67 a b c$ \\
\hline $\mathrm{SL} / \mathrm{P}$ & & & $10 f$ & $11.17 \mathrm{dc}$ & $10.37 a b$ & $8.17 d$ \\
\hline SL/P/R & & & 11.83bcdef & $11.3 \mathrm{dc}$ & $9.33 \mathrm{bc}$ & 8.67bcd \\
\hline $\mathrm{L}$ & & & 10.83cdef & $10.83 \mathrm{~cd}$ & & \\
\hline $\mathrm{L} / \mathrm{P}$ & & & 10.83cdef & $11.5 \mathrm{dc}$ & & \\
\hline $\mathrm{S}$ & & & & & $6.5 d$ & $6.33 e$ \\
\hline SCL/P/R & 21.33fdge & 18.33defg & $12.33 \mathrm{bcde}$ & 12.bcd & $11.33 a b$ & $9.3 a b$ \\
\hline L/R & & & $15 a$ & $12.07 a b c$ & & \\
\hline SCL/R & 18.33fge & $16.67 \mathrm{fg}$ & 12.67bcd & $12.33 \mathrm{bcd}$ & $11.33 a b$ & $10.5 a$ \\
\hline SCL/P & 19.0defg & $15.67 \mathrm{~g}$ & 10.83cdef & $11 \mathrm{~cd}$ & $9.33 \mathrm{bc}$ & $7.5 \mathrm{de}$ \\
\hline $\mathrm{C} / \mathrm{P}$ & $22.0 \mathrm{~cd}$ & $21.67 \mathrm{~g}$ & & & & \\
\hline $\mathrm{C} / \mathrm{P} / \mathrm{R}$ & $26.17 a b$ & $26.33 a$ & & & & \\
\hline $\mathrm{SC}$ & $16.67 \mathrm{~g}$ & 18.defg & & & $10.5 a b$ & $8.33 \mathrm{~cd}$ \\
\hline SC/R & 17.9 & 11defg & & & $12 a$ & 9.67abc \\
\hline $\mathrm{SC} / \mathrm{P}$ & $17.33 \mathrm{fg}$ & $16 \mathrm{~g}$ & & & $8.17 \mathrm{~cd}$ & $8.17 d$ \\
\hline $\mathrm{S} / \mathrm{P}$ & & & & & $9.67 \mathrm{bc}$ & $7.67 \mathrm{de}$ \\
\hline $\mathrm{C}$ & 21.3cde & 20.17dce & & & & \\
\hline $\mathrm{CL} / \mathrm{P}$ & $23.5 a b$ & 19.5cdef & $13 a b c$ & $11.67 \mathrm{dc}$ & & \\
\hline $\mathrm{CL}$ & 20.5 cdef & $21.17 \mathrm{bcd}$ & 12.17bcdef & $10.83 \mathrm{~cd}$ & & \\
\hline $\mathrm{C} / \mathrm{R}$ & $27.67 a$ & $21.67 \mathrm{bc}$ & & & & \\
\hline CL/R & $23.35 b c$ & $24 a b$ & $13.67 \mathrm{ab}$ & $13.67 \mathrm{ab}$ & & \\
\hline CL/P/R & $28.33 a$ & $22.67 \mathrm{bc}$ & $13 a b c$ & $14.33 a$ & & \\
\hline SE \pm & 26 & 0.44 & 0.17 & 0.14 & 0.16 & 0.12 \\
\hline $\mathrm{CV}$ & 8.5 & 0.69 & 9.6 & 8 & 10.1 & 8.8 \\
\hline
\end{tabular}

Means with the same letter (s) within each column are not significantly different at 0.05 level using Duncan's Multiple Range Test.

Legend: $\mathrm{C}=$ Clitoria, $\mathrm{L}=$ lablab, $\mathrm{S}=$ sorghum, $\mathrm{P}=$ phosphors, $\mathrm{R}=$ Rhizobium 
Agric. Biol. J. N. Am., 2011, 2(1): 150-162

Table 4b. Effect of intercropping, phosphorus and inoculation on crude protein (\%) of Clitoria, lablab and sorghum (2006)

\begin{tabular}{|c|c|c|c|c|c|c|}
\hline Treatment & \multicolumn{2}{|l|}{ Clitoria } & \multicolumn{2}{|l|}{ Lablab } & \multicolumn{2}{|l|}{ Sorghum } \\
\hline $\mathrm{L} / \mathrm{P} / \mathrm{R}$ & & & $17.08 \mathrm{a}$ & 11.83abcd & & \\
\hline SC/P/R & $16.08 \mathrm{e}$ & 16.92cdef & & & $10.5 d$ & 8.7defg \\
\hline $\mathrm{SL}$ & & & $9.58 b$ & $9.83 e$ & $10.17 \mathrm{ab}$ & 8.5degf \\
\hline SL /R & & & 10.17gh & $10 \mathrm{e}$ & $9 b c$ & $9.5 \mathrm{bcd}$ \\
\hline $\mathrm{L}$ & & & $10.5 \mathrm{gh}$ & 11.33cde & & \\
\hline $\mathrm{L} / \mathrm{P}$ & & & 10.gh & 10.5 & & \\
\hline $\mathrm{S}$ & & & & & $9.75 d$ & $6.75 b$ \\
\hline SCL/P/R & 16.67 & $20.25 b$ & $11.25 \mathrm{efg}$ & 11.2ced & $10.5 a b$ & $11.5 a$ \\
\hline L/R & & & $14.75 b$ & $13.17 a$ & & \\
\hline SCL/R & $16.42 \mathrm{e}$ & $19.92 b c$ & $10.92 \mathrm{fgh}$ & $13 a b$ & $10.33 a b$ & $9.5 \mathrm{bcd}$ \\
\hline SC/R & $21.17 \mathrm{~cd}$ & 17.83bcef & & & $10.58 \mathrm{a}$ & 8.83cdef \\
\hline $\mathrm{SC} / \mathrm{P}$ & $16.58 \mathrm{e}$ & $15.67 \mathrm{ef}$ & & & $7.42 d$ & 7.83befg \\
\hline $\mathrm{S} / \mathrm{P}$ & & & & & $7.08 d$ & $7.17 \mathrm{gh}$ \\
\hline C & $19.5 d$ & 18.92bcd & & & & \\
\hline CL/P & $19.42 d$ & 18.42bcde & 12.33def & $11.33 \mathrm{cde}$ & & \\
\hline $\mathrm{CL}$ & $19.25 d$ & 17.83bcdef & 12.17ef & $11.67 \mathrm{bcd}$ & & \\
\hline $\mathrm{C} / \mathrm{R}$ & $22.42 b c$ & $24.0 a$ & & & & \\
\hline CL/R & $23.67 a b$ & $19.83 \mathrm{bcd}$ & $13.92 \mathrm{bc}$ & $11.5 \mathrm{~cd}$ & & \\
\hline $\mathrm{CL} / \mathrm{P} / \mathrm{R}$ & $23.75 a b$ & 1.17bcdef & 13.83bcd & 12.50ab & & \\
\hline SE \pm & 0.18 & 0.23 & 0.12 & 0.11 & 0.12 & 0.11 \\
\hline $\mathrm{CV}$ & 6.7 & 8.9 & 7.2 & 6.9 & 9.2 & 8.4 \\
\hline
\end{tabular}

Means with the same letter (s) within each column are not significantly different at 0.05 level using Duncan's Multiple Range Test.

Legend: $\mathrm{C}=$ Clitoria, $\mathrm{L}=$ lablab, $\mathrm{S}=$ sorghum, $\mathrm{P}=$ phosphors, $\mathrm{R}=$ Rhizobium

\section{DISCUSSION}

Individual Yield : Individual yield of sole Clitoria and sole lablab treated with $\mathrm{P}$ and $\mathrm{R}$ was the highest one. This is expected for these leguminous forages as there were less competition and less shading resulting from the cereal forage (Sorghum) when grown as sole crops. However, these forage legumes when intercropped with cereal forages their total yield exceeded that of each crop grown alone as a result of the mutual benefits provided by each component to the other ( nitrogen fixation provided by the leguminous component and $\mathrm{CHO}$ and shelter provided by the cereal component). For the majority of treatments, the sole crops produced higher yield, but sole crop treated with $\mathrm{P}+\mathrm{R}$ gave relative higher dry weight which may reflect the role $P$ in dry matter formation and the Rhizobium in provision of nitrogen ( Reddy et al. ,2003).
Total Yield: Total yield in the intercropping system is more reliable than the individual yield, because it considered all plants per unit area. This study showed that intercropping treatments basically legume to legume combination resulted in higher forage yield which indicated mutual cooperation (khair, 1999), whereas sole sorghum and its combination treatments produced lower yield. Sorghum is a cereal plant, not a nitrogen fixer, but $\mathrm{N}$ consumer, thus these characteristics could affect the final yield of sorghum as a sole crop or in the intercropping system. In addition to the intercropping, application of $P$ plus $R$ inoculation was found to increase total yield in this study, which reflected the contribution of $\mathrm{P}$ in stem elongation and developing of new leaves, whereas inoculation resulted in more nodulation, which help in the process of $\mathrm{N}$ fixation ( Zahran,1999; Kiers et al. ,2008). 
Agric. Biol. J. N. Am., 2011, 2(1): 150-162

Table 5a. Effect of intercropping, phosphorus and inoculation on crude fiber (\%) of Clitoria, lablab and sorghum (2005)

\begin{tabular}{|c|c|c|c|c|c|c|}
\hline \multirow[t]{2}{*}{ Treatment } & \multicolumn{2}{|l|}{ Clitoria } & \multicolumn{2}{|l|}{ Lablab } & \multicolumn{2}{|l|}{ Sorghum } \\
\hline & At $1^{\text {st }}$ harvest & At $2^{\text {nd }}$ harvest & At $1^{\text {st }}$ harvest & At $2^{\text {nd }}$ harvest & At $1^{\text {st }}$ harvest & At $2^{\text {nd }}$ harvest \\
\hline $\mathrm{L} / \mathrm{P} / \mathrm{R}$ & & & $30.48 c$ & $36.84 \mathrm{bcd}$ & & \\
\hline $\mathrm{SCL}$ & 29.27dcef & 32.73ab & 38.47ab & 38.99abc & & \\
\hline$S C / P / R$ & $33.27 a b c$ & $26.33 f$ & & & 40.80ab & $40.33 a b$ \\
\hline $\mathrm{SL}$ & & & $39.53 a$ & $41.30 \mathrm{a}$ & $35.94 c$ & 34.51dce \\
\hline SL /R & & & $30.97 c$ & 34.94de & $40.7 \mathrm{abc}$ & $35.6 \mathrm{dc}$ \\
\hline SL/P & & & $32.78 \mathrm{c}$ & 39.48ab & $39.05 a b c$ & $30.87 \mathrm{gf}$ \\
\hline SL/P/R & & & $31.27 \mathrm{c}$ & $36.74 \mathrm{bcd}$ & $40.07 a b c$ & 39.58ab \\
\hline $\mathrm{L}$ & & & $39.33 a$ & $40.53 a$ & $38.67 a b c$ & 3.02def \\
\hline L/P & & & 37.80ab & $40.37 a$ & & \\
\hline $\mathrm{S}$ & & & & & 40.15 & 40.62ab \\
\hline SCL/P/R & $27.76 \mathrm{ef}$ & 27.44def & $30.50 c$ & 36.89de & $36.6 b$ & $30.24 \mathrm{~g}$ \\
\hline L/R & & & 30.66 & 34.89de & & \\
\hline SCL/R & $26.78 f$ & $27.37 \mathrm{ef}$ & $30.83 c$ & $32.77 \mathrm{e}$ & $39.32 a b c$ & $31.17 \mathrm{gfe}$ \\
\hline SCL/P & 30.07abcdfe & $30.47 \mathrm{bcd}$ & $32.84 \mathrm{c}$ & 39.83ab & $38.03 a b c$ & 35.01de \\
\hline $\mathrm{C} / \mathrm{P}$ & $34.75 f$ & $31.67 a b$ & & & & \\
\hline $\mathrm{C} / \mathrm{P} / \mathrm{R}$ & $25.75 f$ & $28.2 \mathrm{bcde}$ & & & & \\
\hline SC & $33.18 a b c$ & $30.93 a b c$ & & & $38.68 a b c$ & $39.73 a b$ \\
\hline $\mathrm{SC} / \mathrm{R}$ & 27.98def & $30.93 a b c$ & & & $39.7 a b c$ & $37.64 b c$ \\
\hline $\mathrm{SC} / \mathrm{P}$ & 29.71bcdef & $32.12 a b$ & & & $41.96 a$ & $39.5 a b$ \\
\hline $\mathrm{S} / \mathrm{P}$ & & & & & 42.2 & $41.68 \mathrm{a}$ \\
\hline C & $33.75 a b$ & $33.78 a$ & & & & \\
\hline $\mathrm{CL} / \mathrm{P}$ & 32.33abcd & 32.ab & $36.48 \mathrm{~b}$ & 39.24ab & & \\
\hline $\mathrm{CL}$ & 32.0abcd & $31.32 \mathrm{ab}$ & $36.18 b$ & $41.67 \mathrm{a}$ & & \\
\hline $\mathrm{C} / \mathrm{R}$ & 27.93def & 27.89def & & & & \\
\hline $\mathrm{CL} / \mathrm{R}$ & 31.35abcde & 27.9def & $31.62 \mathrm{c}$ & 33.94de & & \\
\hline $\mathrm{CL} / \mathrm{P} / \mathrm{R}$ & $26.03 f$ & 26.94 & $30.89 c$ & $31.91 \mathrm{e}$ & & \\
\hline SE \pm & 0.3 & 0.23 & 0.22 & 0.24 & 0.35 & 0.27 \\
\hline $\mathrm{CV}$ & 7.67 & 5.41 & 4.59 & 4.5 & 6.23 & 5.22 \\
\hline
\end{tabular}

Means with the same letter (s) within each column are not significantly different at 0.05 level using Duncan's Multiple Range Test. Legend: $\mathrm{C}=$ Clitoria, $\mathrm{L}=$ lablab, $\mathrm{S}=$ sorghum, $\mathrm{P}=$ phosphors, $\mathrm{R}=$ Rhizobium 
Agric. Biol. J. N. Am., 2011, 2(1): 150-162

Table 5b. Effect of intercropping, phosphorus and inoculation on crude fiber (\%) of Clitoria, lablab and sorghum (2006)

\begin{tabular}{|c|c|c|c|c|c|c|}
\hline \multirow[t]{2}{*}{ Treatment } & \multicolumn{2}{|l|}{ Clitoria } & \multicolumn{2}{|l|}{ Lablab } & \multicolumn{2}{|l|}{ Sorghum } \\
\hline & At $1^{\text {st }}$ harvest & At $2^{\text {nd }}$ harvest & At $1^{\text {st }}$ harvest & At $2^{\text {nd }}$ harvest & At $1^{\text {st }}$ harvest & At $2^{\text {nd }}$ harvest \\
\hline $\mathrm{L} / \mathrm{P} / \mathrm{R}$ & & & $32.3 b c$ & $32.2 \mathrm{abc}$ & & \\
\hline SCL & $30.15 b c d$ & $29.83 a b$ & $38.7 a$ & 29.3bcde & $40.07 \mathrm{bc}$ & $40.48 a$ \\
\hline $\mathrm{SC} / \mathrm{P} / \mathrm{R}$ & $29.24 d$ & 25.53de & & & $37.17 \mathrm{de}$ & $32.9 d$ \\
\hline $\mathrm{SL}$ & & & $38.5 a$ & 29.4 bcde & $40.22 b$ & \\
\hline $\mathrm{SL} / \mathrm{R}$ & & & $30.8 b$ & 26.31 & $32.52 \mathrm{cde}$ & \\
\hline SL/P & & & $36.6 a$ & $34.0 a$ & $40.9 b$ & \\
\hline SL/P/R & & & $30.2 \mathrm{c}$ & 27.51de & 40.7 & \\
\hline$L$ & & & $39.08 a$ & $29.6 \mathrm{bcd}$ & & \\
\hline $\mathrm{L} / \mathrm{P}$ & & & $36.8 a$ & 28.9dce & & \\
\hline$S$ & & & & & $41.5 b$ & $41.03 a$ \\
\hline SCL/P/R & $27.3 e$ & $27.78 \mathrm{bcd}$ & $30.3 c$ & 27.96de & 37.06 & $39.5 a b$ \\
\hline L/R & & & $30.6 c$ & 26.85 & & \\
\hline SCL/R & $25.49 \mathrm{e}$ & $25.05 \mathrm{e}$ & $30.1 c$ & $29.8 b c d e$ & 38.94bcde & $33.5 \mathrm{~cd}$ \\
\hline SCL/P & $29.6 d c$ & 29.44abc & $35.7 a b$ & $31.8 a b c$ & 39.74cde & $40.2 a$ \\
\hline $\mathrm{C} / \mathrm{P}$ & $31.77 a b c$ & 29.43abc & & & & \\
\hline $\mathrm{C} / \mathrm{P} / \mathrm{R}$ & $26.09 \mathrm{e}$ & 25.58de & & & & \\
\hline SC & $32.28 a b$ & $29 a b c$ & & & $40.1 b$ & $39.15 a b$ \\
\hline $\mathrm{SC} / \mathrm{R}$ & $30.02 d c$ & 25.97de & & & $37.36 \mathrm{de}$ & $36.26 \mathrm{bc}$ \\
\hline $\mathrm{SC} / \mathrm{P}$ & $30.06 \mathrm{abcd}$ & $26.95 \mathrm{cde}$ & & & $41.1 b$ & $40.01 a$ \\
\hline $\mathrm{S} / \mathrm{P}$ & & & & & $44.7 a$ & $39.4 a b$ \\
\hline $\mathrm{C}$ & $32.73 a$ & $30.5 a$ & & & & \\
\hline CL/P & $32.17 a b$ & $30.2 \mathrm{ab}$ & $35.9 a$ & $28.88 \mathrm{cde}$ & & \\
\hline$C L$ & 31.38abc & 30.0ab & $35.4 a b$ & $32.54 a b$ & & \\
\hline $\mathrm{C} / \mathrm{R}$ & $27.24 \mathrm{e}$ & $24.3 e$ & & & & \\
\hline CL/R & $25.28 \mathrm{e}$ & 25.95de & $30.9 c$ & 26.74de & & \\
\hline CL/P/R & $25.61 \mathrm{e}$ & 24.8 & $30.6 c$ & $25.76 \mathrm{e}$ & & \\
\hline SE \pm & 0.19 & 0.2 & 1.96 & 0.27 & 0.22 & 0.2 \\
\hline $\mathrm{CV}$ & 4.6 & 0.5 & 5.8 & 6.5 & 3.6 & 34.6 \\
\hline
\end{tabular}

Means with the same letter (s) within each column are not significantly different at 0.05 level using Duncan's Multiple Range Test. Legend: $\mathrm{C}=$ Clitoria, $\mathrm{L}=$ lablab, $\mathrm{S}=$ sorghum, $\mathrm{P}=$ phosphors, $\mathrm{R}=$ Rhizobium

Land Equivalent Ratio: Land Equivalent Ratio (LER) is a parameter used to evaluate the yield in the intercropping system and as a measure for land use efficiency. In the current study, planting of the three crops (Clitoria, lablab and sorghum) at the same time and on the same plot resulted in the high LER value throughout the two seasons, which indicated the importance of intercropping in land use efficiency than mono-cropping. This result is confirmed by Liu and Zhang (2006)

Crude protein: Generally, Rhizobium inoculation and $\mathrm{P}$ addition increased $\mathrm{CP} \%$ of the forage. The suitable
Rhizobium strain resulted in a successful inoculation causing roots to form nodules, the place of nitrogenfixation and amino acid synthesis, which is the building unit of protein. Mpairwe, et al. (2002) found that intercropping forage legumes with cereals generally resulted in fodder with higher $\mathrm{CP}$ content. Similar result was reported by Abbas (2003).

Crude fiber: In the present study, intercropping and addition of $P$ significantly improved forage quality by decreasing the amount of fiber in the plant tissues. Sole plant was more fibrous than the intercropped one and $P$ treated plant revealed lower percentage of crude fiber. This is in agreement with Mpairwe et al. 
(2002) and Abbas (2003) who found that crude fiber was significantly higher in grasses in pure stand compared to legumes and grass -legume mixture, but in other findings addition of $P$ increased $C F$ (Reddy et al., 2003) but this could be related to time of harvest (plant age) rather than phosphorus addition.

\section{SUMMARY AND RECOMMENDATIONS}

Sole crops produced high amount of individual yield, but in the total yield intercropping treatments yielded high tonnage per unit area. Intercropping treatments had a value of LER greater than one.

Rhizobium inoculation and $\mathrm{P}$ addition increased CP\% of the forage. Intercropping and addition of $P$ significantly improved forage quality by decreasing the amount of fiber in the plant tissues.

- Adoption of intercropping farming system, especially in rural areas, will be more effective and profitable. This is because of the lower input cost and advantages of the intercropping.

- To get healthy forage plant with high nutritional value, adoption of intercropping is important.

- Inoculation of legumes with the suitable Rhizobium strain is important for biological fertilization to reduce input cost and clean environment.

- Land equivalent ratio (LER) is a suitable measure to evaluate the total yield in the intercropping system and see how efficient the land resource is used.

\section{REFERENCES}

Abbas, N.B. (2003). Response of grasses and legumes in mixture and pure stand to phosphorus and nitrogen fertilization at El damazin area. M.Sc. (Agric) Thesis, Faculty of Agriculture, University of Khartoum, Sudan.

A.O.A.C.(Association Of Official Analytical Chemists), (1984). Official methods of the analysis of the Association of Official Analysis, Chem. 14th ed. Published by the A.O.A.C Inc. North Nineteenth Street, Suite 210 Arlington. Virginia, 22209-USA.

Earles, Richard (2005).Sustainable Agriculture: An introduction. ATTR Publication (http//:llattra .ncat.org/attar.pub/PDF/sustaginto.pdf).

Graham, P.H. (2008). Ecological of the root-nodule bacteria of legumes. Chapter (2), p. 23-43. In Dilworth, M. J,
James, E. K, Sprent, J. I and Newton, W.E. (eds.) (2008). Nitrogen-fixing Leguminous Symbioses. Nitrogen Fixation: Orgins, Application. Volume 7. Published by Sprinker. 402 pages.

Khair, M. A. M (1999). Principles of forage production in Sudan. $1^{\text {st }}$ ed. Wad Medani, Sudan (in Arabic)

Kiers, E. T, West, S. A and Denison, R. F (2008). Maintaining cooperation in legume-rhizobia symbiosis: Identifying selection pressures and mechanism. Chapter 3, p59-76. In Dilworth, M. J, James, E. K, Sprent, J. I and Newton, W. E (eds.) (2008). Nitrogenfixing Leguminous Symbioses. Nitrogen Fixation: Orgins, Application. Volume 7. Published by Sprinker. 402 pages

Herridge, D.F. (2008). Inoculation technology of legumes. Chapter (4), p 77-115. In Dilworth, M. J. James, E. K, Sprent, J.I. and Newton, W.E. (eds.) (2008). Nitrogenfixing Leguminous Symbioses. Nitrogen Fixation: Orgins, Application. Volume 7. Published by Sprinker. 402 pages

Li, L, Li, S.M, Sun, J.H, Zhou, L. L, Bao, X. G, Zhang, H. G and Fu-Suo Zhang, F. S (2007). Diversity enhances agricultural productivity via rhizosphere phosphorus facilitation on phosphorus-deficient soils. PNAS, 114 (27):11192-11196.

Li L, Yang SC, Li XL, Zhang FS, Christie P. (1999). Interspecific complementary and competitive interactions between intercropped maize and faba bean. Plant and Soil, 212: 105-114.

Liu, Y and Zhang, L (2006). The Quantitative Evaluation of Output Efficiency in Different Cropping Patterns. Agricultural Sciences in China. 5 (2): 98-102

Mead, R and Willey, R. W (1980). The concept of land equivalent ratio and advantages in yields from intercropping. Exp. Agric., 16 (3): 217-228.

Mpairwe, D. R, Sabiiti, E.N, Ummuna, N. N, Tegegne, A and Osuji, P (2002). Effect of intercropping cereal crops with forage legumes and source of nutrients on cereal grain yield and fodder dry matter yields. African Crop Science Journal, 10(1): 81-97

Reddy, B. V. S, Reddy, P. S, Bidinger, F, Blummel, M (2003) Crop management factors influencing yield and quality of crop residues. Field Crops Research, 84: 57-77.

Shambat Meteorological Station (2008). Annual report

Zahran, H.H. (1999). Rhizobium-legume symbiosis and nitrogen fixation under sever conditions and in an arid climate. Microbiology and molecular biology reviews. 968-989. 